\title{
Haematological implications of folate food fortification
}

\author{
J Metz, MB BCh, MD, DSc (Med), FRSSAfr, FRCPath(UK), FCAP(USA), FRCPA(Australia), DSc (Med)(Hon) \\ Jack Metz was formerly Director of the South African Institute for Medical Research and Professor of Pathology (Haematological), \\ School of Pathology, University of the Witwatersrand, Johannesburg, South Africa.
}

\author{
Corresponding author: J Metz (jac1911@optusnet.com.au)
}

\begin{abstract}
Reports from some Western countries indicate that mandatory folate food fortification (FFF) has substantially reduced the prevalence of folate deficiency, leading to calls for folate testing following FFF to be limited to specific indications such as macrocytic anaemia. This is premature for low-income countries, where folate deficiency is predominantly the result of poor intake coupled with the increasing demand in pregnancy. There is also evidence that HIV infection is prejudicial to folate nutrition, and low-income HIV-infected women and their offspring could be among the most susceptible to folate deficiency. In assessing folate nutrition, the value of serum folate has been compromised by FFF, and both serum and red cell folate are necessary for optimal assessment of folate status. Although the limited data available suggest that large-scale masking of vitamin $B_{12}$ deficiency by FFF has not occurred, it has been suggested that $B_{12}$ be incorporated into folate-fortified foods. However, significant $B_{12}$ deficiency is usually due to malabsorption, and physiological doses added to food would be of questionable value because they would not be absorbed. Extensive work, especially randomised clinical trials, must be done before dietary intervention with $B_{12}$ on a national scale can be justified.
\end{abstract}

S Afr Med J 2013;103(12 Suppl 1):978-981. DOI:10.7196/SAMJ.7022

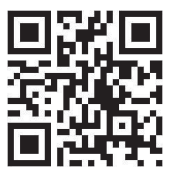

The term folate is used to include both the forms found in natural foods and synthetic folic acid added to fortify foods. Folate-deficient megaloblastic anaemia was recognised in the early 1940s, and was the only known effect of folate deficiency until the 1969 report that folic acid supplementation of pregnant women could reduce the incidence of prematurity. ${ }^{[1]}$ The report ${ }^{[2]}$ by the UK Medical Research Council in 1993 that increasing the folic acid intake of pregnant women reduced the incidence of neural tube defects (NTDs) in their offspring led to the introduction of folate food

It is a privilege to participate in the Festschrift in honour of a longstanding colleague and friend, Professor Trefor Jenkins. The doyen of both modern clinical and population genetics in South Africa, he is unique in having attained international recognition for his contributions to both fields. As the first Professor of Human Genetics at the School of Pathology of the South African Institute for Medical Research and the University of the Wiwatersrand, Johannesburg, he built up an impressive department and trained numerous young workers, many of whom have gone on to assume important positions in the field. A charismatic lecturer and teacher, he expertly communicates his wide knowledge to both students and the lay public. In addition to his outstanding academic achievements, he is a prominent medical ethicist, with a deep concern for human rights and the underprivileged. His numerous efforts to attain justice and equal status for all his colleagues during the apartheid era have been recognised both locally and internationally. He has enriched the lives of all who have been fortunate to know him.

With Professor Jenkins' concern for the underprivileged, it would not seem out of place to devote this paper to the topic of folate food fortification, the benefits of which are aimed primarily at lowincome populations. fortification (FFF) in many countries. In recent years, the literature on FFF has been dominated by its effect on the prevalence of NTDs, and it is perhaps timely to consider some of the haematological implications of FFF.

\section{Historical aspects}

Reports of folate-deficient megaloblastic anaemia in pregnant and lactating women in hospitals in South Africa were published in the early 1950s and led to a number of population surveys of folate nutrition. A high prevalence of deficiency was reported in pregnant and lactating women, non-pregnant women, adult males, older people and preschool children. Deficiency in pregnancy and lactation can be prevented by supplementation with folic acid tablets supplied at antenatal and postnatal clinics, but many pregnant women, particularly in rural areas, did not attend these clinics at that time. Refining of the staple maize meal markedly reduces its folate content, and it was proposed that fortification with folic acid was warranted to combat the widespread deficiency identified. The first studies to test the feasibility of FFF were done in South Africa, completed in 1973 and published by the end of 1975, and have been summarised by Colman. ${ }^{[3]}$ Folic acid added to maize meal, rice or bread cooked in the traditional manner did not alter the colour or taste of the food, and was absorbed adequately. Maize meal with $300 \mu \mathrm{g}, 500 \mu \mathrm{g}$ or $1000 \mu \mathrm{g}$ of folic acid per $30 \mathrm{~g}$ meal added before cooking, and bread with $900 \mu \mathrm{g} / 100 \mathrm{~g}$ added before baking, produced a significant rise in serum and red cell folate concentrations in women in late pregnancy. Maize meal with $300 \mu \mathrm{g}$ folic acid added prevented the progression of folate depletion in late pregnancy. In families given fortified maize meal for use in the home, red cell folate levels rose significantly. These results indicated that fortification of maize meal and bread with folic acid was feasible, and that it was effective in combating folate deficiency. These studies prompted the South African Medical Research Council's Food Fortification Committee to recommend fortification of maize meal with folic acid in 1976. An approach to the milling industry to fortify the meal voluntarily was rejected, and the 
government of the day declined to legislate that the industry replace those vitamins that processing removes so effectively from the meal.

Following the reports of the effect of folic acid in reducing the incidence of NTDs, in 1998 the USA legislated for the mandatory folic acid fortification of flour, cornmeal, rice and pasta in a concentration of $140 \mu \mathrm{g} / 100 \mathrm{~g}$; this example was followed by many other countries, and by 2012 a total of some 66 had instituted or agreed to mandatory FFF, within the range of $100-300 \mu \mathrm{g}$ folic acid per $100 \mathrm{~g}$. The amount of folic acid to be added was designed to increase the folic acid intake to $400 \mu \mathrm{g} /$ day by women who might become pregnant. The South African Government introduced legislation in 2003 for the mandatory fortification of maize meal and wheat flour, at a concentration of $150 \mu \mathrm{g} / 100 \mathrm{~g}$. National programmes to mandate FFF, including that in South Africa, have reduced the prevalence of NTDs significantly worldwide.

\section{Effect of FFF on tests of folate nutrition}

The hallmark of folate deficiency is macrocytic anaemia with megaloblastic change in the bone marrow. Anaemia and macrocytosis, however, are late manifestations and relatively insensitive, nonspecific indicators of deficiency. Measurement of serum folate (SF) and red cell folate (RCF) concentrations enables deficiency to be recognised well before the onset of haematological changes. SF is predominantly a sensitive marker of dietary folate intake, falls rapidly into the deficient range during deprivation, and is the earliest indicator of folate imbalance, before depletion of folate stores. SF is subnormal both when intake is deficient and when body stores are depleted, and does not distinguish between the two. RCF, which reflects folate nutrition at the time of formation of red cells (the folate concentration of which remains constant throughout their 120-day lifespan), is not affected by current folate intake, and is therefore a more specific index of folate nutrition. Folate is required for the conversion of homocysteine (Hcy) to methionine, and Hcy accumulates in the plasma in folate deficiency. Measurement of plasma Hcy is a surrogate test for folate deficiency, but the test is nonspecific as there are numerous other causes of raised Hcy.

The US National Health and Nutrition Examination Survey (NHANES) has monitored the folate status of the US population from pre-fortification to post-fortification over a 12-year period by SF and RCF. Post-fortification SF and RCF were 2.5 times and 1.5 times the pre-fortification concentrations, respectively. The post-fortification prevalence of low SF $(<10 \mathrm{nmol} / \mathrm{l})$ or RCF $(<340 \mathrm{~mol} / \mathrm{l})$ was $<1 \%$, compared with $24 \%$ for SF and $3.5 \%$ for RCC before fortification. ${ }^{[4]}$ A comparable reduction in the prevalence of subnormal folate concentrations after fortification was subsequently reported in population surveys in Canada and other countries that had implemented FFF. At the clinical level, US and Canadian hospitals reported a fall in the number of patients with low folate values following the FFF, with folate-deficient anaemia extremely rare.

The effect of FFF in low-income populations likely to suffer from nutritional deficiencies, and in target groups such as non-pregnant women of reproductive age, pregnant or lactating women, and preschool children, has received some but limited attention. The increase in SF and RCF in the NHANES study occurred regardless of demographic group. In three large county hospitals serving a highly indigent population in the USA, the prevalence of low RCF decreased from $4.8 \%$ in 1997 to $0.65 \%$ after FFF. In Canada, FFF increased the mean RCF from $527 \mathrm{nmol} / 1$ before fortification to $741 \mathrm{nmol} / \mathrm{l}$ after fortification in women of reproductive age.
In an analysis of dietary surveys on adults in South Africa, mean folate intakes were very low, particularly in women, but were raised by FFF to above the recommended nutrient intake. ${ }^{[5]}$ This is reflected in a study of a small sample of non-pregnant, non-lactating women in a rural area in Limpopo Province in which median levels of SF and RCF were significantly increased after FFF, the prevalence of low SF was reduced from $27.6 \%$ to $0 \%$, and the prevalence of low RCF was reduced from $26.4 \%$ to $1.9 \% .{ }^{[6]}$

\section{Testing for folate deficiency in the era of FFF}

Folate testing is currently used in the investigation of patients, particularly those with anaemia, and in population surveys to determine the prevalence of deficiency. In the USA and other high-income countries, the majority of folate tests are done to investigate macrocytic anaemia in non-pregnant patients. Diagnostic laboratories in the USA and Canada have reported a very low prevalence of subnormal SF and RCF in patients post FFF, and this has provoked calls to reduce or eliminate testing for folate deficiency in patients with anaemia and/or macrocytosis. In those countries, folate testing could be limited to specific indications such as macrocytic anaemia with peripheral blood morphological changes suggestive of megaloblastosis, alcoholism, malabsorption syndromes such as coeliac disease (most foods fortified with folic acid are not gluten free), some haemopoietic disorders, and chemotherapy patients. In low-income countries, however, folate deficiency is predominantly the result of poor intake, usually associated with poverty and malnutrition, coupled with the increasing demand in pregnancy; the need for folate testing is therefore much wider, and considerably more data are required before limited folate testing can be justified.

The value of SF as an index of folate intake or nutritional status has been compromised by FFF, and measurement of SF alone is of doubtful clinical value. A recent folate-fortified meal may raise a subnormal SF to normal and thus mask underlying folate deficiency. RCF is not affected by recent intake changes and remains useful in clinical settings. It therefore does not seem unreasonable to suggest that post FFF, RCF alone be used for the detection of folate deficiency. RCF, however, may not detect deficiency of recent onset, for SF falls within 3 weeks of consuming a folate-deficient diet, but RCF only after 120 days. RCF is not entirely specific for folate deficiency, as it may fall also in primary vitamin $B_{12}$ deficiency, despite adequate folate stores. Optimal assessment of folate status after FFF should still include both SF and RCF and also serum $B_{12}$. The extensive assessment of folate nutritional status in populations in the USA as part of NHANES is carried out by measurement of both SF and RCF, and has not changed following the introduction of FFF.

\section{FFF and the implications for patients with vitamin $B_{12}$ deficiency}

The metabolic roles of folate and $\mathrm{B}_{12}$ are inextricably linked. In early reports (1945 - 1948) large doses of folic acid, $5 \mathrm{mg} /$ day or $30 \mathrm{mg}$ 3 times a week, produced haematological remission in patients with pernicious anaemia (PA), the most important result of $B_{12}$ deficiency. In a high proportion of cases the remission was not sustained and neurological relapse was not prevented. In one patient the onset of neurological changes was described as 'explosive', and the rapidity with which neuropathy developed in some patients suggested that folic acid not only failed to prevent neuropathy, but was actively involved in producing it. This led to concern that the administration of folic acid could mask the anaemia and delay the diagnosis of PA, 
allowing neuropathy to develop and progress. While the evidence that folic acid may precipitate neuropathy in patients with $B_{12}$ deficiency is at best debatable, the possibility of masking the anaemia with folic acid provided by FFF merits further consideration.

The doses of folic acid that produced haematological remission in PA patients are far larger than the amount designed by FFF to increase intake to $400 \mu \mathrm{g}$ /day. In general, PA patients do not show haematological response to this dose, but it is possible that no folate supplement can be considered safe if it is to be given to PA patients over a long period of time. ${ }^{[7]}$

The usual finding that alerts to the possibility of $B_{12}$ deficiency is macrocytosis, with or without anaemia, and theoretically this could be masked by the folic acid in fortified foods. There is evidence that this is unlikely, in that the relationship between the serum $B_{12}$ concentration and macrocytosis is not influenced by the serum folate concentration. ${ }^{[8]}$ It would be expected also that following FFF, the number of people with low serum $B_{12}$ but without macrocytosis or anaemia would increase. Reports on this issue are inconsistent. In one study, ${ }^{[9]}$ the proportion of patients with low serum $B_{12}$ but without anaemia did not increase significantly after fortification, but in another the proportion of subjects with low serum $B_{12}$ without macrocytosis was significantly higher after fortification. ${ }^{[10]}$ One disturbing report found that among people with low $\mathrm{B}_{12}$ status in the NHANES, high plasma folate was associated with an increased prevalence of anaemia and cognitive impairment, and an increase in Hcy and methylmalonic acid (an index of $B_{12}$ nutrition); ${ }^{[11]}$ these data imply that high plasma folate is associated with an exacerbation of both the biochemical and clinical status of $\mathrm{B}_{12}$ deficiency.

The limited data available to date suggest that large-scale masking of $B_{12}$ deficiency has not occurred following the implementation of FFF, and after 15 years of mandatory FFF there is no substantial evidence that the higher folic acid intake may harm the public. The possibility of delay in the diagnosis of $B_{12}$ deficiency in patients with PA cannot be entirely discounted, and continued monitoring of possible deleterious effects is mandatory. The possible masking of macrocytosis would be less relevant if clinicians were educated to use the readily available measurement of serum $B_{12}$ for the detection of suspected $\mathrm{B}_{12}$ deficiency, as a significant proportion of PA patients do not show macrocytosis, especially when there is concomitant thalassaemia, iron deficiency, chronic infection, inflammation or marked red cell fragmentation.

To avoid a possible deleterious effect of FFF on undetected $B_{12}$ deficiency, it has been advocated that $B_{12}$ be incorporated into folate-fortified foods. There are, however, very significant differences between the two vitamins. In contrast to folate deficiency, significant $\mathrm{B}_{12}$ deficiency is largely confined to malabsorption, usually due to PA, a disease predominantly of older age groups, and rarely the result of deficient intake, which is seen in vegans. There are large populations in the world subsisting on various combinations of vegetarian diets, with low $B_{12}$ contents, among whom the resultant higher prevalence of lower serum $B_{12}$ concentrations does not translate into an increased prevalence of anaemia. ${ }^{[12]}$ As significant $B_{12}$ deficiency is usually due to malabsorption, physiological doses of $B_{12}$ added to food would be of questionable value in that they would not be absorbed.

The highest prevalence of low serum $B_{12}$ concentrations, with or without an abnormality in $\mathrm{B}_{12}$-dependent biochemical tests, is in older subjects, only a small minority of whom have PA. The low serum $B_{12}$ and/or abnormal biochemical findings are not associated with morbidity in that these people rarely if ever develop haematological or neurological sequelae of $B_{12}$ deficiency. This so-called subclinical $B_{12}$ deficiency is usually attributed to food $B_{12}$ malabsorption, attendant on various degrees of chronic gastritis; low concentrations of the metabolically inert serum $B_{12}$ binder haptocorrin may be a factor in some patients.

Therapeutic trials of oral doses of $B_{12}$ in older people with subclinical $\mathrm{B}_{12}$ deficiency and no evidence of PA have yielded extraordinarily varied and controversial results. With test doses of $10 \mu \mathrm{g}$ and $50 \mu \mathrm{g}$ $B_{12}$ per day, only the $50 \mu \mathrm{g}$ dose produced a significant increase in serum $B_{12}$ concentration. ${ }^{[13]}$ In another study, $600 \mu \mathrm{g} \mathrm{B} B_{12} /$ day, 300 times greater than the recommended dietary allowance of $2 \mu \mathrm{g} /$ day, was needed to normalise low serum $B_{12}$ and raised methylmalonic acid. ${ }^{[14]}$ Results in patients with food $B_{12}$ malabsorption show similar variation, one study showing increased serum $B_{12}$ following a daily dose of $5.9 \mu \mathrm{g},{ }^{[15]}$ while in another $250-1000 \mu \mathrm{g} /$ day was required to have any effect. ${ }^{[16]}$ Although different degrees of chronic gastritis in the patients studied probably contributed to these variable results, it is unlikely to account for the remarkable differences reported. The field becomes even more confused when the studies of the absorption of $B_{12}$ added to food are considered. Breakfast cereal fortified with $4.8 \mu \mathrm{g} \mathrm{B} \mathrm{B}_{12}$ decreased the prevalence of low serum $\mathrm{B}_{12}$ concentrations from $9 \%$ to $3 \%{ }^{[17]}$ and bread with $9.6 \mu \mathrm{g} \mathrm{B}_{12}$ increased the serum $B_{12}$ concentration by $49 \%{ }^{[18]}$ The reason why these relatively small amounts of $B_{12}$ added to food produced an effect, while larger doses without food failed to do so, is not apparent. Patients with PA cannot respond to normal fortification doses, and as would be expected with severe gastric atrophy, doses of $2000-3000 \mu \mathrm{g} /$ day are required to correct the $\mathrm{B}_{12}$ deficiency.

Regardless of the uncertainties about the amount of $B_{12}$ to be added, Carmel ${ }^{[19]}$ has argued that clinical trials to demonstrate actual health benefits of relevant oral doses have not been done in persons with subclinical $B_{12}$ deficiency, who are the only practical targets of $B_{12}$ fortification, because more than $94 \%$ of persons with clinically overt $B_{12}$ deficiency have severe malabsorption. It is only in the severely malabsorptive disorders such as PA, not subclinical deficiency, that neurological deterioration following folic acid therapy has been described. Extensive work, especially randomised clinical trials, must be done before mandatory dietary intervention on a national scale can be justified.

\section{Some further issues relating to FFF in South Africa}

With evidence of widespread folate deficiency in South Africa before the implementation of FFF, the efficacy of FFF in reaching all sections of the population, especially the target groups of pregnant and lactating women and preschool children in low-income groups in rural areas, needs to be determined. The results of the study of a small group of non-pregnant women in a rural area suggests that there is adequate intake of folate-fortified foods, ${ }^{[6]}$ but much more data on target groups are needed. Monitoring of fortification programmes must include accurate assessment of their effect, as well as early detection of possible harm. As mentioned above, the call for reduction or elimination of folate testing of patients following FFF, emanating from high-income countries, is premature for countries in which there are substantial numbers of poor, undernourished people, and the goal of FFF reaching the total population has not been demonstrated.

The impact of the high prevalence of HIV infection in some sections of the population needs to be taken into consideration, for there is evidence that HIV infection is prejudicial to folate nutrition. In a study of lactating HIV-infected and HIV-uninfected women in South Africa, mean folate concentrations were lower in HIV-positive mothers. ${ }^{[20]}$ Similar findings had been reported from Zimbabwe, 
where SF concentrations were lower in HIV-positive women than in uninfected women. The cause of the lower folate concentration is not clear, but it may be related to malabsorption of folate attendant on the changes in the gastrointestinal tract associated with HIV infection. Low-income HIV-infected women in their reproductive years, and their offspring, could therefore be among the most susceptible to folate deficiency.

With regard to the possible masking of $\mathrm{B}_{12}$ deficiency by FFF, in most of the surveys of local adults and children, the prevalence of subnormal serum $B_{12}$ has been low, despite the apparent limited intake of animal protein in low-income groups. There is no greater potential for a harmful effect of FFF in South Africa than anywhere else. It is mainly in patients with severe $B_{12}$ deficiency due to malabsorption, such as PA, that there are concerns regarding the masking of $B_{12}$ deficiency by FFF. There are no data on the prevalence of PA in South Africa, but the disease occurs in all population groups, and the prevalence is probably comparable to that reported worldwide.

A further consideration is the possible effect of increased amounts of dietary folate overcoming the prophylactic and therapeutic effect of antimalarial and chemotherapeutic drugs that act as folate antagonists. This is a particular problem for children in malarial endemic areas, and has led to the World Health Organization warning that supplemental folate should not be given to children in whom folate antagonist antimalarial drugs are used. With the introduction of newer antimalarial drugs with a different mode of action, this should become less of a problem in time.

1. Baumslag N, Edelstein T, Metz J. Reduction of incidence of prematurity by folic acid supplementatio in pregnancy. BMJ 1970;1(5687):16-17. [http://dx.doi.org/10.1136/bmj.1.5687.16]

2. Medical Research Council Vitamin Study Research Group. Prevention of neural tube defects: Results of the Medical Research Council Vitamin Study. Lancet 1991;338(8760):131-137. [http://dx.doi. org/10.1016/0140-6736(91)90133-A]

3. Colman N. Addition of folic acid to staple foods as a selective nutrition intervention strategy. Nutr Rev 1982;40(8):225-233. [http://dx.doi.org/10.1111/j.1753-4887.1982.tb05319.x]

4. Pfeiffer CM, Hughes IP, Lacher DA, et al. Estimation of trends in serum and RBC folate in the US. population from pre- to post-fortification using assay-adjusted data from the NHANES 1988-2010. J Nutr 2012;142(5):886-893. [http:///dx.doi.org/10.3945/jn.111.156919]
5. Steyn NP, Wolmarans P, Nel JH, et al. National fortification of staple foods can make a significant contribution to micronutrient intake of South African adults. Public Health Nutr 2008;11(03):307313. [http://dx.doi.org/10.1017/S136898000700033X]

6. Modjadji SEP, Alberts M, Mambolo RL. Folate and iron status of South African non-pregnant rural women of childbearing age, before and after fortification of foods. South African Journal of Clinical Nutrition 2007;20(3):88-93.

7. Chanarin I. The Megaloblastic Anaemias. 2nd ed. Oxford: Blackwell Scientific Publications, 1979.

8. Metz J, McNeil AR, Levin M. The relationship between serum cobalamin concentration and mean cell volume at varying concentrations of serum folate. Clin Lab Haematol 2004:26(5):323-325. cell volume at varying concentrations of serum fo
[http://dx.doi.org/10.1111/j.1365-2257.2004.00631.x]

9. Mills JL, Von Kohorn I, Conley MR, et al. Low vitamin B-12 concentrations in patients withou anaemia: The effect of folic acid fortification of grain. Am J Clin Nutr 2003;77(6):1474-1477.

10. Wyckoff KF, Ganji V. Proportion of individuals with low serum vitamin B12 concentrations withou macrocytosis is higher in the post folic acid fortification period than in the pre folic acid fortification period. Am J Clin Nutr 2007;86(4):1187-1192.

11. Selhub J, Paul L. Folic acid fortification: Why not vitamin B12 also? Biofactors 2011;37(4):269-271 [http://dx.doi.org/10.1002/biof.173]

12. Metz I. A high prevalence of biochemical evidence of vitamin B12 or folate deficiency does not translate into a comparative prevalence of anaemia. Food Nutr Bull 2008;29(2):S74-S85.

13. Seal EC, Metz J, Flicker L, et al. A randomized, double-blind, placebo-controlled study of oral (a) vitamin B12 supplementation in older patients with subnormal or borderline serum vitamin
B12 concentrations. I Am Geriatr Soc 2002;50(1):146-151. [http://dx.doi.org/10.1046/j.1532B12 concentration

14. Eussen SJ, de Groot LC, Clarke R, et al. Oral cyanocobalamin supplementation in older people with vitamin B12 deficiency: A dose-finding trial. Arch Intern Med 2005;168(10):1167-1172. [http:/ dx.doi.org/10.1001/archinte.165.10.1167]

15. Blacher J, Czernichow S, Raphael M, et al. Very low oral doses of vitamin B-12 increase serun concentrations in elderly subjects with food-bound vitamin B-12 malabsorption. J Nut 2007;137(2):373-378.

16. Andres E, Kaltenbach G, Noblet-Dick M, et al. Efficacy of short-term oral cobalamin therapy for the treatment of cobalamin deficiencies related to food-cobalamin malabsorption: A study of 30 patients. Clin Lab Haematol 2003;25(3):161-166. [http://dx.doi.org/10.1046/j.1365-2257.2003.00515.x]

17. Tucker KL, Olsen B, Bakun GE, et al. Breakfast cereal fortified with folic acid, vitamin B-6, an vitamin $B-12$ increases vitamin concentrations and reduces homocysteine concentrations: $A$ randomized trial. Am J Clin Nutr 2004;79(5):805-811.

18. Winkels RM, Brouwer IA, Clarke R, et al. Bread cofortified with folic acid and vitamin B-12 improved the folate and vitamin B-12 status of healthy older people: A randomized controlled trial. (n)

19. Carmel R. Mandatory fortification of the food supply with cobalamin: An idea whose time has not yet come. J Inherit Metab Dis 2011;34(1):67-73. [http://dx.doi.org/10.1007/s10545-010-9150-2]

20. Papathakis PC, Rollins NC, Chantry CJ, et al. Micronutrient status during lactation in HIV-infected and HIV-uninfected South African women during the first 6 months after delivery. Am J Clin Nut 2007;85(1):182-192.

A full list of references can be obtained from J Metz (jac1911@optusnet.com.au).

Accepted 12 August 2013 